\title{
Leaking false aneurysm of right coronary saphenous vein graft: successful treatment by percutaneous coil embolisation
}

\footnotetext{
Department of Cardiothoracic Surgery, Glasgow Royal Infirmary W R Dimitri

Department of Radiology, Glasgow Royal Infirmary AW Reid

Department of Cardiology, Stobhill Hospital, Glasgow F G Dunn

Correspondence to Mr W R Dimitri FRCS Cardiothoracic Unit, Glasgow Royal Infirmary, Glasgow Royal Infirmary, Glasgow G31 2ER.
}

\begin{abstract}
An unusual complication after aortocoronary bypass grafting (CABG) is described in which a false aneurysm of the saphenous vein graft to the right coronary artery (RCA) developed and caused profuse intermittent bleeding through the sternotomy wound. The aetiology of this condition is uncertain but it could occur whenever a suture line is present especially in the presence of infection. The diagnosis was made non-invasively by a contrast enhanced computed tomogram and was subsequently confirmed by selective coronary bypass angiography. The pseudoaneurysm was successfully obliterated by coil embolisation of the right coronary graft, which stopped the bleeding immediately and was followed by rapid wound healing.
\end{abstract}

(Br Heart J 1992;68:619-20)

Profuse haemorrhage caused by secondary infection of an anastomotic suture line is a well recognised early complication after coronary artery surgery. Less commonly, false aneurysms of the ascending aorta may develop at the site of an aortic incision and present clinically or may be detected at necropsy, though they have seldom been reported in published reports. ${ }^{12}$ False aneurysms related to coronary vein grafts are, however, unusual and difficult to treat. ${ }^{3}$ Because the presentation of these conditions is generally dramatic and the mortality associated with acute rupture is high, immediate surgical intervention has hitherto been the only available treatment, despite being attended with a high morbidity and frequently an unpredictable outcome.

We report a case in which a false aneurysm of the saphenous vein graft to the right coronary artery developed and caused frequent episodes of profuse intermittent bleeding through the sternum with failure of sternal wound healing. As an alternative to surgical management we used emergency coil embolisation of the vein graft, a technique that we believe has not been used before in such a case.

\section{Case report}

A 63 year old man with a long history of angina was investigated in 1989 and found to have extensive triple coronary artery disease with reduction of left ventricular function caused by hypokinesia of the inferior segment. At that time he was also known to have diffuse peripheral vascular disease of both ileofemoral segments and a right profundaplasty had been performed a year before. Coronary artery bypass surgery was undertaken in September 1990 and an internal mammary artery was applied to the left anterior descending coronary artery and vein grafts to the distal right and obtuse marginal vessels. The patient's subsequent progress was complicated initially by major secondary haemorrhage from an infected tear in the ascending aorta after staphylococcal wound infection and sternal dehiscence: the tear was thought to have been a direct consequence of repeated sternal friction and erosion of the right lateral wall which lay close to the vein graft anastomoses. Through a considerably restricted access, the friable area was successfully repaired with a vein patch. The sutures were applied under direct vision. Recurrent sternal wound infections over several weeks, which required close observation, delayed his discharge from hospital. The sternotomy wound seemed to heal slowly and he required readmission after repeated minor episodes of haemorrhage from an area of infected granulation tissue in the middle of the sternotomy incision. Further episodes of minor bleeding often occurred and on one occasion he had a massive lifethreatening haemorrhage.

A dynamic computed tomogram showed a pool of extravascular contrast medium behind and erosion through the inner table of the sternum. A track could be identified that almost certainly originated from the proximal segment of a vein graft. A flase aneurysm from the ascending aorta could not be excluded because of the initial aortic bleed after surgery. Aortography and selective vein graft angiography (fig 1) confirmed the presence of a false aneurysm originating from the proximal third of the graft to the right coronary artery. The distal part of that vessel was still patent though the run off was sluggish. This factor coupled with the presence of hypokinesia of the inferior segment led us to think that occluding this vessel would not cause a significant area of infarction. The possibility of surgical correction was also considered but was thought to carry too high a risk. Embolism of the right coronary artery graft was therefore regarded as the only realistic alternative. 
Figure 1 Selective coronary angiogram of a right coronary vein graft (curved arrow). A large false aneurysm ( $A n)$ arising from the proximal third of the vein graft can be seen adherent to the sternum with

extravasation of contrast medium into the retrosternal region (arrow heads).

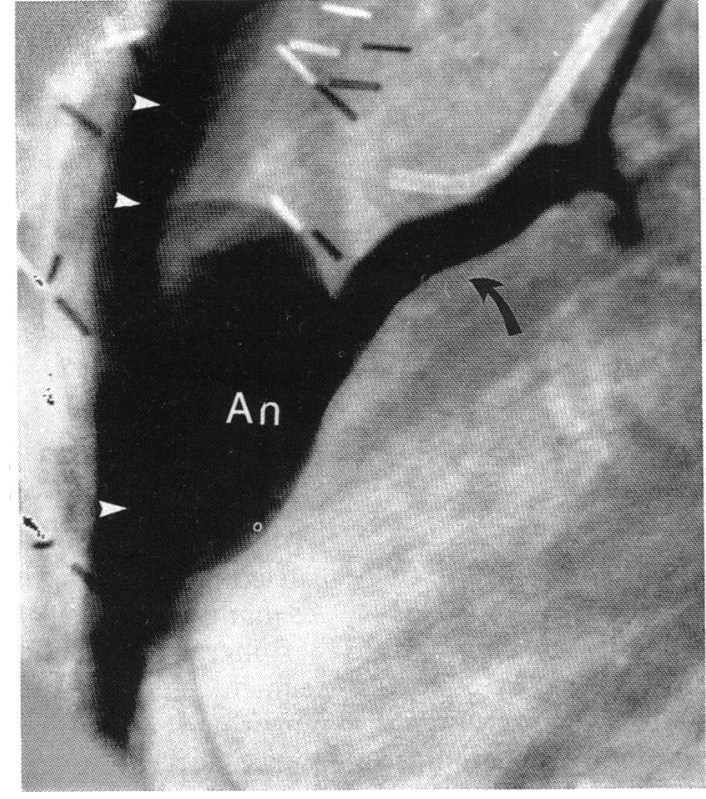

The graft was gauged to be a vessel with a $6 \mathrm{~mm}$ diameter, but at the time of emergency intervention only $5 \mathrm{~mm}$ and $8 \mathrm{~mm}$ coil emboli were available. An $8 \mathrm{~mm}$ coil was placed in the mid portion of the graft but owing to the disparity in size and the turbulent bi-directional flow between the graft and the aneurysm, the coil was dislodged into the systemic circulation and wedged in the left common iliac artery. Fortunately, this proved to be clinically inconsequential. A $5 \mathrm{~mm}$ coil was too small and was carried by the forward flow into the false aneurysm sac. The catheter was advanced to the mouth of the aneurysm and a further $8 \mathrm{~mm}$ coil was placed, straddling the vein graft and the ostium of the false aneurysm to provide a framework for the insertion of further $5 \mathrm{~mm}$ and $3 \mathrm{~mm}$ coils. These reduced flow and subsequently occluded the graft (fig 2 ). Heated contrast medium (iopamidol 370 at $80^{\circ} \mathrm{C}$ ) was also instilled to promote thrombus formation. ${ }^{4}$

The patient tolerated this procedure remarkedly well. The bleeding stopped immediately and the sternal wound healed rapidly over the

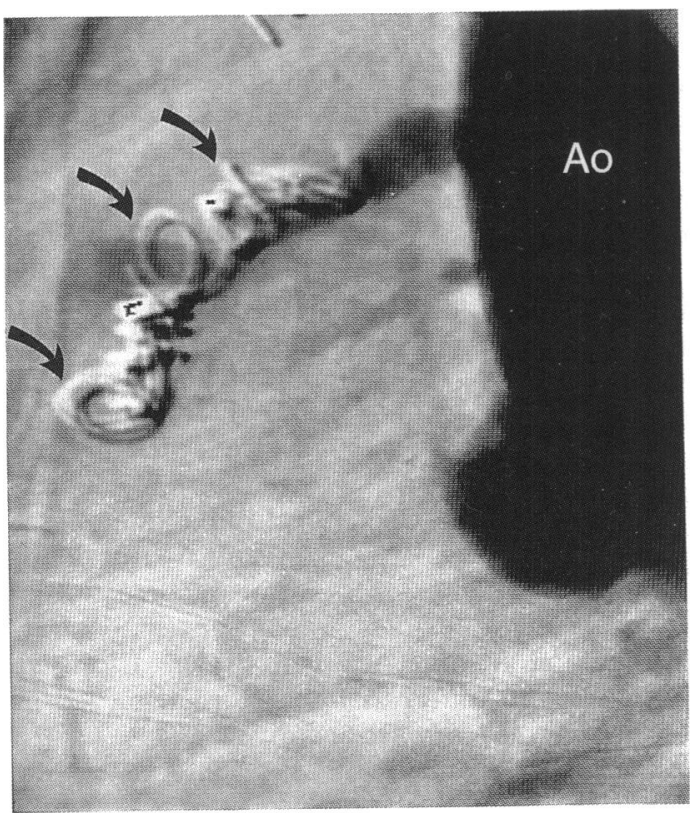

next two weeks (fig 1B). Serial electrocardiograms and enzyme estimations showed no evidence of myocardial damage after occlusion of the right coronary graft. Transient renal dysfunction caused by the contrast medium resolved rapidly within a few days on conservative treatment. The patient continues to do well with adequate peripheral circulation and his claudication distance unchanged.

\section{Discussion}

The occurrence of false aneurysms after aortocoronary surgery is a rare but potentially lethal complication attended with high morbidity that is difficult to treat. Traditionally, the management has been surgical but because successful intervention has seldom been reported, optimal treatment has usually been determined by the personal experience of the clinician. In view of the site of the aneurysm in our patient we believed that it could not be safely managed by further sternotomy: alternative surgical approaches were regarded as equally hazardous. Embolisation of the vein graft feeding the false aneurysm was considered the most appropriate treatment.

Several individual case reports have described false aneurysms arising from the aorta, commonly in relation to a suture line and often as a consequence of secondary infection after operation. In our case, it was clinically unclear whether the aneurysm originated in the aorta or from the vein graft itself. A computed tomogram, however, suggested a vein graft origin and this was conclusively demonstrated by selective angiography.

The only technical difficulties associated with the procedure were related to the lack of suitable coil sizes. It had been estimated angiographically that the vein graft was a $6 \mathrm{~mm}$ diameter vessel but only $5 \mathrm{~mm}$ and $8 \mathrm{~mm}$ coils were available. Nonetheless, profuse bleeding meant that intervention was urgent. Correct sizing and scrupulous placement of these devices are fundamental to the successful obliteration of the vessel. The initial coil must be sufficiently large to wedge against the wall of the conduit and provide a matrix for subsequent coils. The thrombogenic nature of these occluders together with the resultant sluggish flow hold the coils tightly into position until a secure thrombus firmly obliterates the vessel permanently.

Intervention was judged to be successful when a computed tomogram showed occlusion of the vein graft and obliteration of the false aneurysmal sac, bleeding did not recur, and the wound healed rapidly. This technique was clearly better than a surgical approach and we regard embolisation as the best treatment for false aneurysms of vein grafts after coronary artery surgery.

1 Roberts WC. Aortic dissection: Anatomy, consequences and causes. Am Heart J 1981;101:195-214.

2 Saffitz JE, Ganote CE, Paterson CE, Roberts WC. False aneurysm after aortocoronary bypass grafting. $\mathrm{Am}$ Cardiol 1983;52:907-9.

3 Smith P, Qureshi S, Yacoub MH. Dehiscence of infected aortocoronary vein graft suture lines. Cause of late aortocoronary vein graft suture lines. Cause of late pseudoaneury.

4 Rholl KS, Rysavy JA, Vlodaver Z, Cragg AH, CastanedaZuniga WR, Amplatz K. Transcatheter thermal venous occlusion: A new technique. Radiology 1982;145:333-7. 